\title{
Fatores de risco para doença trofoblástica gestacional persistente
}

\author{
Risk factors for persistent gestational trophoblastic disease \\ Daniel Guimarães Tiezzi ${ }^{1}$, Jurandyr Moreira de Andrade², Francisco José Candido dos Reis ${ }^{3}$, \\ Wellington Lombardi ${ }^{4}$, Heitor Ricardo Cosiski Marana ${ }^{5}$
}

\section{RESUMO}

Objetivos: avaliar o impacto dos fatores de riscos na evolução para doença trofoblástica gestacional persistente (DTGP) e selecionar grupos de pacientes para seguimento intensivo e os que poderiam se beneficiar de quimioterapia profilática. Métodos: foram incluídas prospectivamente 214 pacientes com diagnóstico de mola hidatiforme completa (MHC) submetidas a esvaziamento uterino no período de 1980 a 2001 . Todas as pacientes foram seguidas semanalmente com avaliação clínica e dosagem de $\beta$ HCG. Consideramos como DTGP as pacientes que necessitaram tratamento adicional além do esvaziamento uterino para a resolução do caso. Foram analisados parâmetros epidemiológicos (idade, antecedentes obstétricos, raça e tipagem sanguínea) bem como indicadores de volume e agressividade da doença (volume uterino, presença de cistos tecaluteínicos e dosagem sérica de $\beta \mathrm{HCG}$ ). Os diversos fatores de risco foram avaliados isoladamente e em conjunto, sendo o risco expresso em odds ratio (OR). Resultados: dentre os fatores epidemiológicos e características pessoais apenas a ausência do fator $\mathrm{Rh}$ foi significante (com OR de 2,3). Todos os sinais indicativos de hiperplasia do trofoblasto, representados pela altura uterina maior que a esperada para a idade gestacional, o volume uterino estimado pela ultra-sonografia, a presença de cistos teca-luteínicos e a dosagem sérica elevada de $\beta \mathrm{HCG}$, estiveram associados ao risco de DTGP. A presença de pelo menos um destes achados mostrou sensibilidade de $82 \%$ e valor preditivo positivo de $35,1 \%(\mathrm{OR} 4,8)$. A regressão logística identificou os parâmetros altura uterina maior que o esperado para a idade gestacional e os níveis séricos de $\beta \mathrm{HCG}$ como fatores de risco para DTGP com OR de 4,1 e 5,5, respectivamente. Conclusões: os sinais de hiperplasia do trofoblasto apresentam boa sensibilidade na predição de DTGP, no entanto o baixo valor preditivo positivo impede que se empreguem estes fatores para selecionar pacientes que não necessitariam de seguimento na forma realizada atualmente e impede também a seleção com precisão de casos com indicação de quimioterapia profilática.

PALAVRAS-CHAVE: Mola hidatiforme; Neoplasias trofoblásticas gestacionais; Quimioterapia; Fatores de risco

\section{ABSTRACT}

Purpose: to evaluate the epidemiologic data and signs of trophoblastic hyperplasia in patients with complete hydatidiform mole (CHM) and to estimate the risk associated with the persistence of the disease. Methods: we evaluated 214 patients with CHM submitted to uterine evacuation between 1980 and 2001. The patients were included prospectively. All patients were followed until negative $\beta H C G$ with weekly clinical evaluation and $\beta H C G$ quantification. We considered persistence when the patient needed another treatment after uterine evacuation. The risk factors for persistence were evaluated through univariate and multivariate analysis, and the odds ratio (OR) was calculated for each one. Results: among the epidemiologic factors, only negative $\mathrm{Rh}$ was significant $(\mathrm{OR}=2.28)$. All signs of trophoblastic hyperplasia, represented by uterine size larger than expected, sonographic uterine volume, tecaluteinic cysts, and $\beta H C G$ higher than $10^{5}$ were associated with risk for the presistence of the disease. The presence of at least one sign of trophoblastic hyperplasia showed sensitivity of $82 \%$ and predictive positive value of $35.1 \%(\mathrm{OR}=4.8)$. The logistic regression identified larger uterine size than expected and $\beta \mathrm{HCG}$ higher than $10^{5}$ as risk factors for persistence of the gestational trophoblastic disease ( $\mathrm{OR}=4.1$ and 5.5, respectively). Conclusions: the signs of trophoblastic hyperplasia showed good sensitivity to predict persistence of the disease; however, the low predictive positive value does not allow using these criteria to change treatment. It is very important to reinforce the importance of serial $\beta H C G$ quantification in these high-risk patients.

KEYWORDS: Hydatidiform mole; Gestational trophoblastic neoplasms; Drug therapy; Risk factors

1 Médico Assistente - Hospital das Clínicas de Ribeirão Preto - Faculdade de Medicina de Ribeirão Preto - Universidade Estadual de São Paulo - USP Ribeirão Preto (SP) - Brasil.

2 Docente do Departamento de Ginecologia e Obstetrícia - Faculdade de Medicina de Ribeirão Preto - Universidade Estadual de São Paulo - USP Ribeirão Preto (SP) - Brasil.

3 Docente do Departamento de Ginecologia e Obstetrícia - Faculdade de Medicina de Ribeirão Preto - Universidade Estadual de São Paulo - USP Ribeirão Preto (SP) - Brasil.

4 Pós-graduando (Mestrado) - Programa de Tocoginecologia - Universidade Estadual de São Paulo - USP - Ribeirão Preto (SP) - Brasil e Bolsista - Fundação de Amparo à Pesquisa do Estado de São Paulo - FAPESP - São Paulo (SP) - Brasil.

5 Médicos Assistente - Hospital das Clínicas de Ribeirão Preto - Faculdade de Medicina de Ribeirão Preto - Universidade Estadual de São Paulo - USP Ribeirão Preto (SP) - Brasil.

Correspondência: Daniel Guimarães Tiezzi

Hospital das Clínicas da Faculdade de Medicina de Ribeirão Preto - USP - Departamento de Ginecologia e Obstetrícia - Avenida Bandeirantes n 3900 14049-900 - Ribeirão Preto - São Paulo - Telefone: (16) 602-2583 - FAX: (16) 633-0946 - e-mail: daniel_tiezzi@yahoo.com.br

Recebido em: 6/6/2005 Aceito com modificações em: 25/6/2004

Rev Bras Ginecol Obstet. 2005;27(6):331-9 


\section{Introdução}

A mola hidatiforme e a doença trofoblástica gestacional persistente DTGP, (mola invasora e metastática) são pouco freqüentes, mas representam problema importante em termos de saúde reprodutiva pela faixa etária afetada e pela morbidade, mortalidade e pelo risco de comprometimento do potencial reprodutivo como resultado da própria doença ou do seu tratamento ${ }^{1}$. Com freqüência as pacientes são jovens e em cerca de metade dos casos, a mola hidatiforme ocorre na primeira gravidez ${ }^{2}$.

O seguimento adequado após o tratamento inicial da mola hidatiforme por vácuo-aspiração ou curetagem permite o diagnóstico precoce das formas persistentes ${ }^{3}$ e, como conseqüência, há diminuição da freqüência de doença metastática e das formas de alto risco de resistência ao tratamento ${ }^{4}$. A quimioterapia aplicada às formas persistentes, apesar de ser quase sempre efetiva, tem, como conseqüência, além dos custos individuais e sociais, as complicações e o aumento de risco de novas neoplasias ${ }^{5}$.

A porcentagem de pacientes que evoluem para doença persistente após tratamento inicial da mola hidatiforme varia amplamente entre as diferentes casuísticas (7 a $36 \%)^{6-9}$. As taxas mais baixas são relatadas pelos serviços britânicos, com porcentagem de $5,1 \%{ }^{10}$. Esta variação pode indicar diferenças entre as populações, mas deve-se principalmente ao emprego de diferentes critérios para diagnóstico da doença persistente, considerando-se que a mortalidade é semelhante quando se comparam as séries nas quais a proporção de pacientes tratadas com quimioterapia é tão diferente. Assim, é possivel que parte das pacientes esteja recebendo tratamento desnecessário. $\mathrm{Na}$ tentativa de uniformizar as indicações de tratamento, a FIGO criou e divulgou a partir de 2001 novo sistema de diagnóstico e estadiamento ${ }^{11,12}$.

O modelo de seguimento indicado inclui dosagens semanais de gonadotrofina até a negativação e mensais por até um ano, associadas a exame físico periódico. No entanto, altas taxas de abandono são observadas até antes da negativação dos níveis de gonadotrofinas ${ }^{13,4}$, e a porcentagem de pacientes que comparece a todos os retornos oscila entre 18 e $63 \%$. Estas falhas no seguimento, no entanto, não implicam necessariamente aumento da taxa de doença de alto risco ou da mortalidade $^{10}$, e as pacientes que durante o seguimento têm pelo menos um resultado conside- rado negativo têm o risco para doença persistente substancialmente reduzido ${ }^{13,14}$ ou mesmo nulo, se o método de dosagem empregado for de alta sensibilidade $(5 \mathrm{mIU} / \mathrm{mL})^{15}$. Estas observações indicam que seria útil identificar, já no início do seguimento, o grupo de casos com indicadores de maior risco de evolução para doença persistente que deveriam receber assistência diferenciada e seguimento de acordo com os protocolos atuais.

Outro aspecto relacionado à identificação de grupos de risco para DTGP é a indicação de quimioterapia profilática para pacientes com mola hidatiforme. Embora não seja de aceitação ampla, o seu uso é admitido em áreas nas quais as dificuldades para seguimento são maiores ${ }^{16}$ ou para pacientes com menor adesão ao seguimento ${ }^{17}$. No entanto, se aplicada ao conjunto das pacientes com mola sem seleção, os benefícios não são signifi$\operatorname{cativos}^{18}$. Com a aplicação de critérios clínicos e epidemiológicos é possivel selecionar um grupo restrito de mais alto risco e que é beneficiado ${ }^{19}$.

Portanto, para selecionar pacientes que necessitam de seguimento mais intensivo, ou para as quais a quimioterapia profilática estaria indicada, é necessária a identificação de um grupo de maior risco de doença persistente. Os critérios que têm sido utilizados para esta identificação são numerosos e heterogêneos, variando desde indicadores epidemiológicos a características das pacientes, como o tipo sanguíneo e $\mathrm{Rh}^{20}$, os ligados ao tipo histológico e à hiperplasia do trofoblasto $^{21}$ e niveis circulantes de várias substâncias ${ }^{22}$. Outros indicadores como a reduzida atividade apoptótica ${ }^{23}$, a expressão de oncogenes, marcadores de proliferação e os relacionados ao controle do ciclo celular como as ciclinas D e $\mathrm{E}^{24}$, têm sido estudados mas os resultados ainda não podem ser empregados, devido ao pequeno número de casos incluídos nos estudos.

$\mathrm{O}$ indicador de risco mais importante para desenvolvimento de doença persistente ou metastática é o tipo da mola, sendo muito mais elevado para os casos com mola hidatiforme completa em comparação aos que apresentam mola parcial ou habitada ${ }^{1}$. Para os casos de mola parcial as taxas de doença persistente são de 2 a $5 \%{ }^{25}$. Outros critérios histopatológicos, como anaplasia e proliferação do trofoblasto ${ }^{26}$, não se mostraram relevantes ${ }^{27}$, e atualmente considera-se como indicador histológico de alto risco, o diagnóstico de coriocarcinoma ${ }^{11}$. Entre os fatores epidemiológicos mais estudados estão: a idade ${ }^{28}$, raça ${ }^{29}$, história reprodutiva pregressa ${ }^{30}$ e grupo sanguíneo ${ }^{20}$. 
Os indicadores clínicos estão relacionados ao volume da mola hidatiforme, que por sua vez depende da taxa de proliferação do trofoblasto. Entre estes indicadores podemos citar: volume uterino grande para a idade gestacional, altos níveis de gonadotrofinas circulantes e seus efeitos: cistos teca-luteínicos e outras complicações como hipertiroidismo. Em algumas séries estes parâmetros podem indicar de 25 até $100 \%$ dos casos que apresentarão persistência ${ }^{31}$.

Com o objetivo de verificar o impacto dos fatores epidemiológicos e dos sinais de hiperplasia do trofoblasto na evolução das pacientes com o diagnóstico de mola hidatiforme completa, e verificar a acurácia destes indicadores de risco, desenvolvemos o presente estudo.

Métodos

Foram incluídas no estudo 214 pacientes com o diagnóstico de mola hidatiforme completa, atendidas consecutivamente no período de 1980 a dezembro de 2001. Todas haviam sido admitidas vindas de serviços de atendimento primário ou de outros hospitais da região para esvaziamento de mola ou curetagem para abortamento. Durante este período, foram atendidas no Hospital das Clínicas da Faculdade de Medicina de Ribeirão Preto da USP 416 pacientes com doença trofoblástica, das quais 148 haviam sido encaminhadas para seguimento após esvaziamento da mola ou já com diagnóstico de doença persistente. Foram também atendidas oito pacientes com diagnóstico de doença trofoblástica após gravidez a termo (parte dos casos publicados em outro estudo), dois após gravidez ectópica e 44 casos com diagnóstico histológico de mola parcial.

Todas as pacientes incluídas foram submetidas a esvaziamento uterino no serviço. Os casos com diagnóstico clínico ou ultra-sonográfico de abortamento foram tratados inicialmente com curetagem e os casos com diagnóstico ultrasonográfico de doença trofoblástica gestacional foram submetidos à aspiração a vácuo. O material obtido foi encaminhado para análise histopatológica e todos os casos incluídos tinham diagnóstico histológico de mola hidatiforme completa ${ }^{26}$. Após o esvaziamento as pacientes foram mantidas em seguimento ambulatorial semanal com exame fisico e avaliação semiquantitativa dos niveis urinários de gonadotrofina (período de 1980 a 1989) ou dosagem sérica da subunidade $\beta$ da gonadotrofina coriônica (de 1990 a 2001) por quimiluminescência (DPC - Immunlite) até a negativação, e então a periodicidade passava a ser mensal por até seis meses. Se a resolução era espontânea a paciente recebia alta após seis meses e se necessitasse de quimioterapia, o seguimento após a negativação era de um ano.

As duas categorias (mola invasora e doença metastática) foram agrupadas sob a denominação de DTGP, ao passo que as formas com resolução espontânea foram denominadas como doença trofoblástica gestacional benigna (DTGB). A distinção entre DTGP e DTGB foi baseada na evolução clínica do caso e, principalmente, na evolução da dosagem sérica ou urinária da fração beta da gonadotrofina coriônica humana ${ }^{11}$.

Do grupo de 214 pacientes, 155 apresentaram resolução espontânea (DTGB) e 59 (27,5\%) evoluíram com DTGP. Foram classificadas como DTGB as pacientes que apresentaram regressão dos niveis séricos da subunidade beta de gonadotrofina coriônica até a sua negativação, além da remissão completa de eventuais sinais clínicos apenas com o esvaziamento do conteúdo uterino. Como DTGP foram classificadas as pacientes que necessitaram de tratamento adjuvante (quimioterapia ou cirurgia) durante o seguimento pós-esvaziamento. O tratamento adjuvante foi indicado com base na evolução das dosagens séricas da subunidade beta: aumento dos niveis de gonadotrofinas por duas semanas consecutivas ou pela ocorrência de platô por três semanas ou mais. Nenhum caso apresentou diagnóstico histológico de coriocarcinoma e entre estes casos não houve detecção de metástases sem elevação prévia dos niveis de gonadotrofinas. Nenhuma paciente foi submetida a histerectomia antes do diagnóstico de doença persistente.

Compareceram a todos os retornos agendados 53\% das pacientes; deixaram de comparecer antes de completar três dosagens negativas em três semanas consecutivas $8,1 \%$ dos casos e, entre eles, somente dois não freqüentaram os retornos até pelo menos um resultado negativo. Um destes casos apresentou posteriormente doença metastática de alto risco. Os casos que deixaram de comparecer foram contactados por assistente social, de forma que informações sobre a evolução puderam ser obtidas para todos os casos. As pacientes tratadas com quimioterapia foram seguidas conforme rotina do serviço por pelo menos um ano após o término do tratamento.

Foram analisados como potenciais indicadores de risco para desenvolvimento de doença 
persistente alguns parâmetros epidemiológicos como idade e antecedentes gestacionais: número de gestações prévias e a sua evolução - aborto ou gravidez a termo. A classificação dos casos quanto à raça (branca e não branca) foi feita por funcionários treinados do serviço de registro. O tipo de sangue foi classificado em $A, B, A B$ e $O$ e, para fins de análise, as pacientes foram divididas conforme apresentassem ou não o antígeno A (comparação entre tipos $\mathrm{O}$ e B contra A e AB). Foram obtidas durante o período de inclusão a classificação do sangue em Rh positivo ou negativo e esta variável foi incluída no estudo.

Como indicadores do volume e agressividade da doença foram pesquisadas e analisadas as relações entre o volume uterino obtido clinicamente e a idade gestacional (menor, igual ou maior que o esperado). Outros indicadores de agressividade foram o volume uterino estimado pela ultra-sonografia $\left(\mathrm{em}^{\mathrm{c}} \mathrm{cm}^{3}\right)$, a presença de cistos teca-luteínicos e a dosagem da subunidade beta no soro, prévia ao esvaziamento uterino. Estes fatores foram considerados em conjunto como indicadores de hiperplasia do trofoblasto.

Os casos com diagnóstico de doença persistente foram estadiados na fase de tabulação dos dados de acordo com o proposto pela FIGO em 2001, inclusive quanto ao escore de risco ${ }^{11,32}$. As informações sobre as pacientes foram coletadas em formulários individuais que incluem todas as informações relevantes sobre os atendimentos e evolução dos casos. Para este trabalho as informações foram coletadas destes formulários e arquivadas em banco de dados construído com o software Microsoft Access.

Os indicadores de risco para DTGP foram avaliados com o uso de análise estatística univariada. Foi empregado o teste exato de Fisher para estimativa do odds ratio (OR). Para comparação das médias de idade, da idade gestacional e volume uterino estimado pela ultra-sonografia entre os grupos DTGB e DTGP foi utilizado o teste $t$ de Student. Para comparação das medianas dos niveis séricos de $\beta \mathrm{HCG}$ entre os grupos de DTGB e DTGP foi utilizado teste $t$ não paramétrico de MannWhitney. A análise multivariada dos fatores independentes foi realizada por meio de regressão logística. Foi considerado estatisticamente significante $\mathrm{p}<0,05$. Foram elaboradas curvas ROC dos volumes uterinos e das dosagens séricas do $\beta \mathrm{HCG}$ para obtenção do melhor ponto de corte como indicador de risco para DTGP.

\section{Resultados}

A mediana da idade foi de 23 anos; 54 pacientes tinham idade inferior a 20 anos $(25,2 \%)$ e 23 tinham idade superior a 35 anos (10,7\%). A DTGP ocorreu em 16 pacientes com idade inferior a 20 anos $(29,6 \%)$ e em seis pacientes com idade superior a 35 anos (26\%). A média da idade das pacientes com DTGB foi de 24,5 anos (faixa de variação de 13 a 49) e das pacientes com DTGP, de 25,6 anos (15 a 50). Estas diferenças não foram significantes (teste $t$ de Student; $\mathrm{p}=0,35)$.

Quanto à raça, 84,0\% das pacientes eram brancas (apenas uma da raça amarela) e na comparação com as não brancas, não se observou diferença na distribuição por raça entre as pacientes com DTGB e DTGP $(85,1$ e $81,3 \%$ de pacientes brancas, respectivamente - teste exato de Fisher; $p=0,53)$. Os antecedentes obstétricos analisados foram: história de abortos espontâneos (teste exato de Fisher; $p=0,39)$ e antecedente de gestações a termo (teste exato de Fisher; $p=0,75$ ). Nenhum destes antecedentes, portanto, pôde discriminar entre as pacientes com mola que evoluíram para DTGP.

O tipo sanguíneo pôde ser verificado em 152 pacientes. Foram classificadas em dois grupos, conforme apresentassem ou não o antígeno A. Não encontramos associação da presença deste antígeno com evolução para DTGP (teste exato de Fisher; $p=0,35)$. Já a ausência do fator Rh foi associada a risco significante para o desenvolvimento de doença persistente (teste exato de Fisher; $\mathrm{p}=0,038 \mathrm{com}$ OR=2,28; IC 95\% de 1,07 a 4,8). A Tabela 1 resume os resultados obtidos na análise univariada dos fatores epidemiológicos.

Tabela 1 - Fatores epidemiológicos indicativos de risco para o desenvolvimento de doença trofoblástica gestacional persistente - análise univariada.

\begin{tabular}{lcccc}
\hline & DTGB & DTGP & $\mathbf{p}$ & OR \\
\hline Idade (média em anos) & 24,5 & 25,6 & 0,35 & NS \\
Raça branca (\%) & 85,1 & 81,3 & 0,53 & NS \\
Gestação a termo prévia (\%) & 60,6 & 57,6 & 0,75 & NS \\
Abortos prévios (\%) & 25,8 & 32,2 & 0,39 & NS \\
Presença do antígeno A (\%) & 50,6 & 58,6 & 0,35 & NS \\
Fator Rh negativo (\%) & 12,9 & 24,1 & 0,0038 & 2,28 (1,07 a 4,8)
\end{tabular}

DTGB - doença trofoblástica gestacional benigna.

DTGP - doença trofoblástica gestacional persistente.

$\mathrm{OR}$ - odds ratio. 
Em relação à idade gestacional no momento do diagnóstico, obtivemos dados confiáveis em 142 dos 155 casos de DTGB e 48 dos 59 de DTGP. Nos casos de DTGB, a média da idade gestacional foi 13,8 semanas (6 a 28), e nos casos de DTGP, a média foi de 13,0 semanas (4 a 20) (teste $t$ de Student; $p=0,31$ ).

Foi possivel avaliar a relação entre a altura uterina obtida por mensuração clínica e a esperada pela idade gestacional em 178 pacientes. Nas 78 pacientes com altura uterina maior que o esperado para a idade gestacional, 33 (42\%) desenvolveram doença persistente. A DTGP ocorreu em apenas $14 \%$ das pacientes com altura uterina considerada compativel com a idade gestacional. A proporção de casos com altura uterina maior que o esperado para a idade gestacional foi maior entre as pacientes que desenvolveram doença persistente (teste exato de Fisher; $\mathrm{p}<0,0001 ; \mathrm{OR}=4,5$; IC $95 \%$ de 2,18 a 9,27).

Informações precisas sobre o volume uterino estimado pela ultra-sonografia foram obtidas de 85 casos de DTGB e 32 casos de DTGP. A média foi de $611,8 \mathrm{~cm}^{3}$ (variação de 68 a $1473 \mathrm{~cm}^{3}$ ) entre as pacientes com DTGB e 856,7 cm3 (187 a 2223) para o grupo que evoluiu para DTGP (teste $t$ de Student; $p=0,0045)$. A elaboração da curva ROC apresentou como melhor ponto de corte o valor $569 \mathrm{~cm}^{3}$. Utilizando este ponto de corte, no entanto, o volume uterino estimado pela ultra-sonografia não demonstrou ser indicador de risco (teste exato de Fisher; $p=0,09$ ).

Em 91 casos de DTGB e 17 de DTGP, foi possível a avaliação ultra-sonográfica dos ovários. Foram encontrados cistos teça-luteínicos em 38,4\% das DTGB e 62,9\% das DTGP na época do esvaziamento ou durante o seguimento, antecedendo o diagnóstico de doença persistente. (teste exato de Fisher; $p=0,028$; OR=2,7; IC 95\% de 1,11 to $6,61)$.

Foi possivel a avaliação dos niveis de $\beta \mathrm{HCG}$ prévios ao esvaziamento uterino em 74 casos de DTGB e 36 casos de DTGP; a mediana nos casos de DTGB foi 89.500 mUI (0 a 3.657.000) e nos casos de DTGP, 224.800 mUI (4800 a $512.600)$, sendo esta diferença significante (teste de Mann-Whitney; $p=0,0056)$. A elaboração da curva ROC de sensibilidade e especificidade dá como melhor ponto de corte o valor 106.000U/mL. Utilizando-se ponto de corte com $100.000 \mathrm{mIU} / \mathrm{mL}$, a dosagem sérica do $\beta \mathrm{HCG}$ pré-esvaziamento mostrou ser um indicador de risco (teste exato de Fisher; $\mathrm{p}=0,01 ; \mathrm{OR}=2,8$; IC $95 \%$ de 1,2 a 6,8$)$.
A presença de pelo menos um sinal de hiperplasia do trofoblasto entre os avaliados em análise univariada (volume uterino maior que o esperado para a idade gestacional, nível do $\beta$ HCG sérico antes do esvaziamento e presença de cisto teca-luteínicos) pôde ser avaliada em 198 pacientes entre as quais 54 pacientes apresentaram DTGP $(27,3 \%)$. Em 118 pacientes com a presença de pelo menos um sinal de hiperplasia do trofoblasto, a DTGP foi diagnosticada em 45 pacientes $(38,1 \%)$. Em 80 pacientes sem sinais de hiperplasia do trofoblasto, apenas nove desenvolveram DTGP $(11,2 \%)$. O achado de pelo menos um sinal de hiperplasia do trofoblasto está associado com maior risco de desenvolvimento de doença persistente (teste exato de Fisher; $\mathrm{p}<0,0001 ; \mathrm{OR}=4,8$; IC 95\% de 2,2 a 10,6). A Tabela 2 resume os resultados obtidos com a análise univariada dos fatores associados à hiperplasia do trofoblasto.

Tabela 2 - Indicadores de risco para o desenvolvimento de doença trofoblástica gestacional persistente relacionados à hiperplasia do trofoblasto - análise univariada.

\begin{tabular}{lcccc}
\hline & DTGB & DTGP & p & OR (IC 95\%) \\
\hline $\begin{array}{l}\text { Volume uterino maior que } \\
\text { o TA (\%) }\end{array}$ & 34,5 & 70,2 & $<0,0001$ & 4,5 (2,18 a 9,27) \\
$\begin{array}{l}\text { Média do volume uterino } \\
\text { (ultra-sonografia em cm }{ }^{3} \text { ) }\end{array}$ & 611,8 & 856,7 & 0,09 & NS \\
$\begin{array}{l}\text { Presença de cistos teça- } \\
\text { luteínicos (\%) }\end{array}$ & 38,4 & 62,9 & 0,028 & $2,72(1,11$ a 6,61) \\
$\begin{array}{l}\text { PHCG pré-esvaziamento } \\
\text { (mediana em mUI) }\end{array}$ & 89.500 & 224.800 & 0,0056 & $2,9(1,2$ a 6,8)
\end{tabular}

DTGB - doença trofoblástica gestacional benigna.

DTGP - doença trofoblástica gestacional persistente.

TA - tempo de amenorréia.

$\mathrm{OR}$ - odds ratio.

IC $95 \%$ - intervalo de confiança a $95 \%$.

As variáveis independentes que se mostraram relevantes pela análise univariada foram, portanto: volume uterino maior que o esperado para a idade gestacional, nível do $\beta$ HCG sérico antes do esvaziamento superior a $100.000 \mathrm{mUI} /$ $\mathrm{mL}$, presença de cisto teca-luteínicos e ausência do fator Rh. A análise por regressão logística das variáveis independentes pode de ser efetuada em 58 casos e demonstrou que apenas a presença do volume uterino maior que o esperado para a idade gestacional ( $p=0,03$; OR=4, 1 ; IC 95\% de 1,1 a 14,8$)$ e os niveis séricos do $\beta \mathrm{HCG}(\mathrm{p}=0,02 ; \mathrm{OR}=5,5$; IC $95 \%$ de 1,3 a 23,8). Os resultados da regressão logística estão resumidos na Tabela 3. 
Tabela 3 - Indicadores de risco para o desenvolvimento de doença trofoblástica gestacional persistente relacionados à hiperplasia do trofoblasto - análise univariada.

\begin{tabular}{lcc}
\hline & p & OR (IC 95\%) \\
\hline Fator Rh negativo (\%) & 0,62 & $0,63(0,1$ a 3,8) \\
Volume uterino maior que o esperado (\%) & 0,03 & $4,1(1,1$ a 14,7) \\
Presença de cistos teça-Iuteínicos (\%) & 0,09 & $2,9(0,83$ a 10,6) \\
bHCG pré-esvaziamento (mediana em mUI) & 0,002 & $5,5(1,3$ a 23,8) \\
\hline
\end{tabular}

OR - odds ratio.

IC $95 \%$ - intervalo de confiança a $95 \%$.

\section{Discussão}

A porcentagem de casos com mola hidatiforme que evoluem para formas persistentes é muito variável, indo de 7 a 35\%. Esta variação depende de vários fatores como características individuais, mas parece ser dependente dos critérios empregados para diagnóstico e da taxa de adesão ao seguimento. Em nosso estudo a porcentagem de casos com doença persistente $(27,5 \%)$ é mais alta em relação a algumas séries que apresentam taxas em torno de $15 \%{ }^{15}$, mas comparável à de outras, com taxas entre $26,4^{27} \mathrm{e}$ $28 \%{ }^{13}$. A taxa de evasão foi muito baixa $(8 \%)$ e informações sobre a evolução foram obtidas prospectivamente para todos os casos, o que permite confiabilidade ao avaliar o potencial dos fatores estudados como indicador de risco de evolução para DTGP.

O modelo de seguimento para pacientes com doença trofoblástica gestacional que inclui dosagens semanais de gonadotrofina até a negativação e mensais por pelo menos seis meses associadas a exame físico periódico tem sido desafiado de várias formas. Por um lado, muitas séries publicadas mostram altas taxas de abandono até mesmo antes da negativação dos niveis de gonadotrofinas. As taxas de abandono variam de $3^{13}$ a $13 \%{ }^{4}$. No entanto, a falta de adesão a formas recomendadas de seguimento não implicam necessariamente aumento da taxa de doença de alto risco ou da mortalidade ${ }^{10}$. Além disto tem se observado que as pacientes que durante o seguimento têm pelo menos um resultado considerado negativo têm o risco para doença persistente substancialmente reduzido ${ }^{13}$ ou mesmo nulo, se o método de dosagem empregado é de alta sensibilidade $(5 \mathrm{mUI} / \mathrm{mL})^{15}$. Estas observações indicam que seria útil identificar já no início do seguimento o grupo de casos com indicadores de maior risco de evolução para doença persistente pas-

sivel de receber assistência diferenciada e seguimento mais longo.

Outro aspecto relacionado à identificação de grupos de risco para DTGP é a indicação de quimioterapia profilática para pacientes com mola hidatiforme. Embora não seja de aceitação ampla, o seu uso é admitido em áreas nas quais as dificuldades para seguimento são maiores ${ }^{16}$ ou para pacientes com menor adesão ao seguimento ${ }^{17}$. Os resultados do uso da quimioterapia profilática, no entanto, não são homogêneos, se aplicada ao conjunto da pacientes com mola, sem seleção, os benefícios não são significativos. Portanto, é indispensável que o eventual grupo a ser tratado seja definido com precisão para evitar que pacientes sejam expostas às complicações que ocorrem em cerca de $25 \%$ dos casos $^{18}$.

Considerando os diversos indicadores isoladamente, observamos que a idade e antecedentes de abortamento não são indicadores de risco para doença persistente, o que também é sugerido em outros estudos com casuísticas menores ${ }^{21}$. No entanto, a análise em conjunto das séries em que os efeitos da idade são analisados mostra que este fator é relevante ${ }^{33}$. Em uma destas séries a porcentagem de doença persistente foi quase três vezes maior para as mulheres com mais de 50 anos comparadas às com 15 anos ou menos $(37,5$ e $13,9 \%$, respectivamente ${ }^{28}$ ). Em nosso estudo, não ocorreu doença trofoblástica gestacional em pacientes com mais de 50 anos, e esta pode ser a razão de não termos encontrado influência da idade na persistência da doença trofoblástica gestacional.

Os dados publicados a respeito do antecedente de abortamento, como indicador de risco, por outro lado, são claramente insuficientes para conclusões ${ }^{24}$. Em nosso estudo, nem a história de abortamentos anteriores aumentou o risco para persistência nem a história de gestações a termo conferiu proteção. No entanto, esta observação não é conclusiva, pois provavelmente a associação está relacionada à idade, visto que o número de gestações é dependente desta ${ }^{34}$.

Algumas características pessoais, como o grupo sangüíneo e a raça, têm sido associados à persistência da neoplasia trofoblástica gestacional. Quanto à influência do tipo de sangue na evolução das pacientes, as informações publicadas são muito heterogêneas. Enquanto alguns encontraram risco de persistência associado aos tipos $\mathrm{O}, \mathrm{B}$ e $\mathrm{AB}^{35}$, em outro estudo o risco estimado foi de 2,2 para mulheres com tipo A e 4,8 para $\mathrm{AB}^{20}$. No entanto, esta associação foi res- 
trita às pacientes com 35 anos ou mais. Nenhum dos tipos do sistema ABO pôde ser associado com risco maior para DTGP. Por outro lado, as pacientes $\mathrm{Rh}$ negativas apresentaram maior risco para doença persistente. Quanto à raça, em nosso estudo não houve diferença quando se comparou a raça branca com a não branca. Nossos resultados demonstram que os fatores epidemiológicos e características pessoais são capazes de detectar apenas $22,8 \%$ das pacientes com evolução para DTGP, não sendo, portanto, passiveis de aplicação clínica.

O outro conjunto de indicadores de risco é relacionado à hiperplasia do trofoblasto: volume uterino maior que o esperado pela idade gestacional, níveis elevados de gonadotrofinas coriônicas, presença de cistos teca-luteínicos e complicações como hipertiroidismo e pré-eclâmpsia. A sua relevância é confirmada pela inclusão em todos os escores de risco. Na nossa casuística todos eles, isoladamente, mostraram associação com a evolução para DTGP.

Para avaliação do volume molar empregamos dois critérios: a medida da altura uterina e sua relação com a idade gestacional e a medida direta do volume uterino obtida pela ultrasonografia. A presença de altura uterina maior que a esperada foi capaz de indicar $70,2 \%$ dos casos que evoluíram para DTGP, com $34,4 \%$ de falsospositivos (OR de 4,5). Ao contrário, o volume uterino estimado pela ultra-sonografia não se mostrou indicativo de risco para DTGP. Esta divergência entre dois critérios para avaliação da mesma variável poderia ser superada pela inclusão de maior número de casos. Os cistos teca-luteínicos, resultantes da hiperestimulação dos ovários pela gonadotrofina coriônica, foram encontrados em porção significativamente superior nos casos que evoluíram para DTGP e a OR associada a este critério foi de 2,7 .

O nivel de gonadotrofinas circulantes é o critério mais freqüentemente associado a risco de evolução para DTGP e, com freqüência, o único critério relevante ${ }^{36}$. O valor mais empregado é de $100.000 \mathrm{mUI} / \mathrm{mL}^{37}$. Este valor pode ser considerado baixo em relação aos observados por nós, pois a mediana dos valores pré-esvaziamento foi de $89.500 \mathrm{mUI} / \mathrm{mL}$ para as pacientes que evoluíram com resolução espontânea e de 224.800 mUI/mL para o grupo com DTGP. Entre nossos casos o risco de evoluir para DTGP com niveis pré-esvaziamento de $\beta \mathrm{HCG}$ superiores a $100.000 \mathrm{mUI} / \mathrm{mL}$ foi de 2,9 .
A avaliação em conjunto dos fatores de risco independentes relacionados com a doença trofoblástica persistente demonstrou que apenas dois fatores, volume uterino maior que o esperado para a idade gestacional e os niveis séricos $\beta \mathrm{HCG}$, estão associados com risco elevado de DTGP, e o risco de evolução é de 4,1 e 5,5, respectivamente.

Concluímos que os melhores indicadores de risco considerando a sensibilidade, especificidade e custos para obtenção das informações são os relacionados à hiperplasia do trofoblasto. Entre eles, a altura uterina aumentada em relação à idade gestacional e os níveis pré-esvaziamento da subunidade beta da gonadotrofina coriônica são os melhores indicadores. No entanto, em função do baixo valor preditivo positivo $(35,1 \%)$ estes critérios não são suficientes para justificar alterações na forma atual de seguimento ou indicação de tratamento quimioterápico profilático. No entanto, as pacientes com indicadores de mais alto risco devem ser especialmente estimuladas a freqüentar os retornos e permanecer em seguimento. Em situações em que o seguimento é problemático, a quimioterapia profilática poderia ser considerada. No entanto, estas situações seriam excepcionais, tendo em vista o custo desta abordagem e as potenciais seqüelas desta forma de tratamento em mulheres jovens e sem prole constituída.

\section{Referências}

1. Smith HO. Gestational trophoblastic disease epidemiology and trends. Clin Obstet Gynecol. 2003;46(3):541-56.

2. Mungan T, Kuscu E, Dabakoglu T, Senoz S, Ugur M, Cobanoglu O. Hydatidiform mole: clinical analysis of 310 patients. Int J Gynaecol Obstet. 1996;52(3):2336.

3. Uberti EM, Diestel MC, Guimaraes FE, Goloubkova T, Rosa MW, De Napoli G. Gestational trophoblastic disease: one more risk in adolescent pregnancy. Acta Obstet Gynecol Scand. 2002;81(4):356-63.

4. Massad LS, Abu-Rustum NR, Lee SS, Renta V. Poor compliance with postmolar surveillance and treatment protocols by indigent women. Obstet Gynecol. 2000;96(6):940-4. 
5. Ng TY, Wong LC. Diagnosis and management of gestational trophoblastic neoplasia. Best Pract Res Clin Obstet Gynaecol. 2003;17(6):893-903.

6. Bagshawe KD, Dent J, Webb J. Hydatidiform mole in England and Wales 1973-83. Lancet. 1986;2(8508):673-7.

7. Soper JT. Management of gestational trophoblastic disease. Oncology (Williston Park). 1993;7(11):6874,82 .

8. Lurain JR, Brewer JI, Torok EE, Halpern B. Natural history of hydatidiform mole after primary evacuation. Am J Obstet Gynecol. 1983;145(5):591-5.

9. Nakano R, Sasaki K, Yamoto M, Hata $H$. Trophoblastic disease: analysis of 342 patients. Gynecol Obstet Invest. 1980;11(4):237-42.

10.Pisal N, Tidy J, Hancock B. Gestational trophoblastic disease: is intensive follow up essential in all women? BJOG. 2004;111(12):144951.

11. Hancock BW. Staging and classification of gestational trophoblastic disease. Best Pract Res Clin Obstet Gynaecol. 2003;17(6):869-83.

12. Soper JT. Staging and evaluation of gestational trophoblastic disease. Clin Obstet Gynecol. 2003;46(3):570-8.

13. Feltmate CM, Batorfi J, Fulop V, Goldstein DP, Doszpod J, Berkowitz RS. Human chorionic gonadotropin follow-up in patients with molar pregnancy: a time for reevaluation. Obstet Gynecol. 2003;101(4):732-6.

14.Batorfi J, Vegh G, Szepesi J, Szigetvari I, Doszpod J, Fulop V. How long should patients be followed after molar pregnancy? Analysis of serum HCG follow-up data. Eur J Obstet Gynecol Reprod Biol. 2004;112(1):95-7.

15. Wolfberg AJ, Feltmate C, Goldstein DP, Berkowitz RS, Lieberman E. Low risk of relapse after achieving undetectable HCG levels in women with complete molar pregnancy. Obstet Gynecol. 2004;104(3):551-4.

16. Sivanesaratnam V. Management of gestational trophoblastic disease in developing countries. Best Pract Res Clin Obstet Gynaecol. 2003; 17(6):925-42.

17.Khoo SK. Clinical aspects of gestational trophoblastic disease: a review based partly on 25year experience of a statewide registry. Aust $\mathrm{N} \mathrm{Z} \mathrm{J}$ Obstet Gynaecol. 2003;43(4):280-9.

18. Kashimura Y, Kashimura M, Sugimori H, Tsukamoto N, Matsuyama T, Matsukuma K, et al. Prophylactic chemotherapy for hydatidiform mole.
Five to 15 years follow-up. Cancer. 1986;58(3):6249.

19. Limpongsanurak S. Prophylactic actinomycin D for high-risk complete hydatidiform mole. J Reprod Med. 2001;46(2):110-6.

20. Parazzini F, La Vecchia C, Franceschi S, Pampallona S, Decarli A, Mangili G, et al. ABO blood-groups and the risk of gestational trophoblastic disease. Tumori. 1985;71(2):123-6.

21. Murad TM, Longley JV, Lurain JR, Brewer JI. Hydatidiform mole: clinicopathologic associations with the development of postevacuation trophoblastic disease. Int $\mathrm{J}$ Gynaecol Obstet. 1990;32(4):359-67.

22. Hegab HM, Schindler AE. The prognostic value of serum inhibin, 17 beta-estradiol and progesterone in cases of hydatidiform mole. Gynecol Endocrinol. 2004;18(2):107-13.

23. Fong PY, Xue WC, Ngan HY, Chan KY, Khoo US, Tsao SW, et al. Mcl-1 expression in gestational trophoblastic disease correlates with clinical outcome: a differential expression study. Cancer. 2005;103(2):268-76.

24. Olvera M, Harris S, Amezcua CA, McCourty A, Rezk $\mathrm{S}$, Koo $\mathrm{C}$, et al. Immunohistochemical expression of cell cycle proteins E2F-1, Cdk-2, Cyclin E, p27(kip1), and Ki-67 in normal placenta and gestational trophoblastic disease. Mod Pathol. 2001;14(10):1036-42.

25. Bracken MB, Brinton LA, Hayashi K. Epidemiology of hydatidiform mole and choriocarcinoma. Epidemiol Rev. 1984;6:52-75.

26. Szulman AE, Surti U. The syndromes of hydatidiform mole. II. Morphologic evolution of the complete and partial mole. Am J Obstet Gynecol. 1978;132(1):207.

27. Morrow CP, Kletzky OA, Disaia PJ, Townsend DE, Mishell DR, Nakamura RM. Clinical and laboratory correlates of molar pregnancy and trophoblastic disease. Am J Obstet Gynecol. 1977;128(4):42430.

28. Bandy LC, Clarke-Pearson DL, Hammond CB. Malignant potential of gestational trophoblastic disease at the extreme ages of reproductive life. Obstet Gynecol. 1984;64(3):395-9.

29. Brinton LA, Bracken MB, Connelly RR. Choriocarcinoma incidence in the United States. Am J Epidemiol. 1986;123(6):1094-100.

30. Parazzini F, La Vecchia C, Pampallona S, Franceschi $\mathrm{S}$. Reproductive patterns and the risk of gestational trophoblastic disease. Am J Obstet Gynecol. 1985;152(7 Pt 1):866-70. 
31.Parazzini F, Mangili G, Belloni C, La Vecchia C, Liati $\mathrm{P}$, Marabini R. The problem of identification of prognostic factors for persistent trophoblastic disease. Gynecol Oncol. 1988;30(1):57-62.

32. Benedet JL, Bender H, Jones H 3rd, Ngan HY, Pecorelli S. FIGO staging classifications and clinical practice guidelines in the management of gynecologic cancers. FIGO Committee on Gynecologic Oncology. Int J Gynaecol Obstet. 2000;70(2):209-62.

33. Altieri A, Franceschi S, Ferlay J, Smith J, La Vecchia C. Epidemiology and aetiology of gestational trophoblastic diseases. Lancet Oncol. 2003;4(11):670-8.
34. Steigrad SJ. Epidemiology of gestational trophoblastic diseases. Best Pract Res Clin Obstet Gynaecol. 2003;17(6):837-47.

35. Sasaki K, Hata H, Nakano R. ABO blood group in patients with malignant trophoblastic disease. Gynecol Obstet Invest. 1985;20(1):23-6.

36. Ayhan A, Tuncer ZS, Halilzade H, Kucukali T. Predictors of persistent disease in women with complete hydatidiform mole. J Reprod Med. 1996;41(8):591-4.

37. Kohorn EI. The new FIGO 2000 staging and risk factor scoring system for gestational trophoblastic disease: description and critical assessment. Int $\mathrm{J}$ Gynecol Cancer. 2001;11(1):73-7. 